\title{
Wireless transport protocol variants for cognitive radio networks
}

\author{
G Shine Let*, G Josemin Bala, W. Magdalene \\ Department of Electronics and Communication Engineering, Karunya University, \\ Karunya Nagar, Coimbatore 64114, India \\ *Corresponding author, e-mail: shinelet@gmail.com
}

\begin{abstract}
Scarce wireless resources, lead to development of cognitive radio network as a solution to unlicensed users communication in the licensed frequency band. In response to the behavior of licensed users communication, unlicensed users communication need to change from one frequency band to another band. In this communication paradigm, the performance of unlicensed users transmission control protocol gets degraded due to the features of cognitive radio network. To overcome this, several authors suggested quite a few modifications in the existing wireless transport protocol for cognitive radio network environment. This paper gives an overview of different transport protocols used for unlicensed users' communication in cognitive radio networks.
\end{abstract}

Keywords: cognitive radio network, transport protocol, congestion window

Copyright $₫ 2018$ APTIKOM - All rights reserved.

\section{Introduction}

Cognitive Radio technology is introduced by Joseph Mitola III in 1999 to have sophistication in the radio layer of communicating devices [1]. This is an intelligent radio which can change its transmission parameters based on the available environment conditions. Cognitive radio is built on top of Software-Defined Radio (SDR) [1]. SDR technology has been implemented in various DSP and general purpose processors for varying coding techniques based on the processing of data, voice, image etc. Intelligence added to SDR is called Cognitive Radio. Cognitive Radio progression into network started in the year 2006. A device which is having communication and networking capability added with cognitive radio features is called Cognitive Radio (CR) node. A network consisting of CR nodes is called Cognitive Radio Network (CRN) [2]. As per the analysis done by FCC, 700MHz spectrum band allotted for Television broadcasting have not been utilized in fullest manner [3]. The UHF band $(300 \mathrm{MHz}$ to $3 \mathrm{GHz})$ is called 'sweetspot', which is highly utilized for various applications such as GSM phones, 3G phones, Wifi, WiMax, Mobile TV, Digital TV, etc., and the number of users are overcrowded. As years go by, finding a frequency band for new users in sweetspot spectrum is difficult. To overcome this spectrum scarcity, CR node can be used for various communication purposes and it can operate in different spectrum bands based on the availability of spectrum.

Primary Users or licensed users (PUs) are users where fixed licensed frequency band is allotted for their communication. The users having CR nodes will able to use the licensed spectrum band, when the primary users are not utilizing it. These users are called Secondary Users (SUs) or Cognitive Users (CUs) or unlicensed users [2]. PUs have guaranteed Quality-of-Service (QoS) for their communication and CUs are provided with best-effort QoS. When the PU arrives, CU has to vacate that spectrum band and move to another spectrum band [4-5]. Due to shifting of spectrum band, the end-to-end communication gets disturbed. The transport protocol has to maintain the end-to-end communication without any packet loss or connection drop. The main functions of transport protocol are congestion control and flow control. The flow control mechanism helps the sender to transmit the number of packets based on the buffer size and the destination packet processing capability. Mostly, based on destination feedback flow control will be adjusted. Also based on the congestion in the network, the number of packets transmitted should be adjusted. In cognitive radio network, due to spectrum sensing and spectrum change, the end-to-end connection get disconnected [2] and the QoS parameters get affected. Many authors have implemented different algorithms to improve the QoS of SUs communication. This paper gives an overview of TCP on wireless networks, different protocols implemented in wireless network to maintain end-to-end semantics, 
discussion of different algorithms implemented and the research issues and challenges of TCP in Cognitive radio network.

\section{TCP Protocols Used in Wireless Network}

When the communications moved from wired to wireless, the main characteristics that affected the wireless network are channel contention, signal fading, mobility and power limitations [6]. The wireless network considered can be infrastructure or infrastructureless (Adhoc). Due to these characteristics, TCP performance has suffered because of segment loss and packet reordering. Many TCP protocols are implemented to provide solutions based on congestion detection approach, state suspension approach, response postponement approach and hybrid approach [7]. For congestion control in the network, TCP Tahoe, TCP Reno, TCP Vegas and TCP SACK protocols are implemented. Based on different application of data transmission a variation on the above protocols was done for communication.

Congestion Detection Approach is used to detect the packet loss due to network congestion, so congestion window is adjusted based on packet loss. Various protocols are implemented to reduce the packet loss due to congestion are TCP-Peach, TCP-Probing, TCP Westwood, TCP-Jersey and TCPCasablanca. The general Congestion window adjustment [7] is shown in Figure1. Based on the above protocol, the quantities represented by ' $*$ ' is varied.

Based on the current-state network condition, end-to-end connection is suspended and resumed to avoid segment loss in state suspension approach. Freeze-TCP, Interlayer collaboration (ILC)-TCP, TCPFeedback, Explicit Link Failure Notification (ELFN)-TCP and TCP-DOOR are the state suspension approaches. In response postponement approach, TCP client delays the communication to alleviate the problems in wireless networks. Various proposed techniques are Delayed ACK TCP, TCP- Adaptive Delayed Acknowledgement (ADA) and TCP-Delayed Congestion Response (DCR). Adhoc-TCP (ATCP) is based on hybrid approach and is implemented for infrastructureless wireless network.

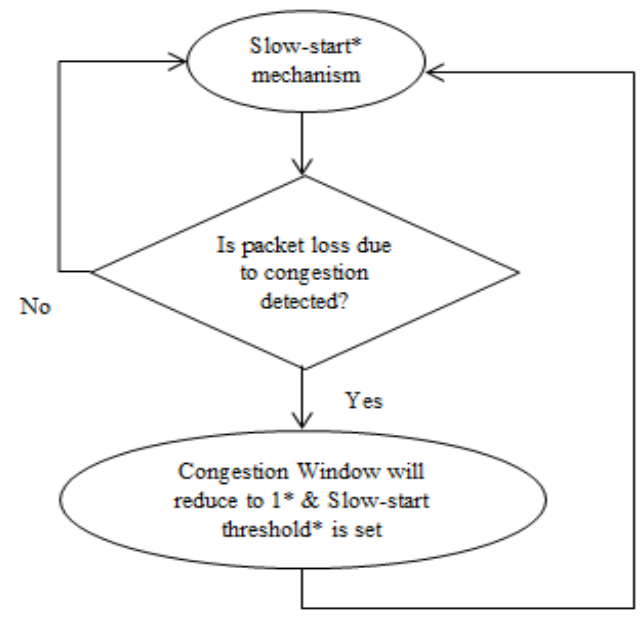

Figure 1. Effects of selecting different switching under dynamic condition

\section{Necessity of Improvement in CRN TCP Protocols}

In cognitive radio network, end-to-end communication between users gets disturbed due to different characteristics in the CR network. The main functions of each node in CR network are shown in Figure 2 [8]. The performance of the TCP in CRN varies due to specific reasons such as PU behavior, Spectrum sensing and Spectrum changing.

\subsection{PU Behaviour}

Primary User is a licensed user gets highest priority in communication. While the secondary user communication is going on, as the PU arrives, secondary user has to vacate that frequency band and move to another vacant frequency band. This result in large amount of packet loss and the performance of TCP is affected. More number of retransmissions takes place due to PU behavior. 


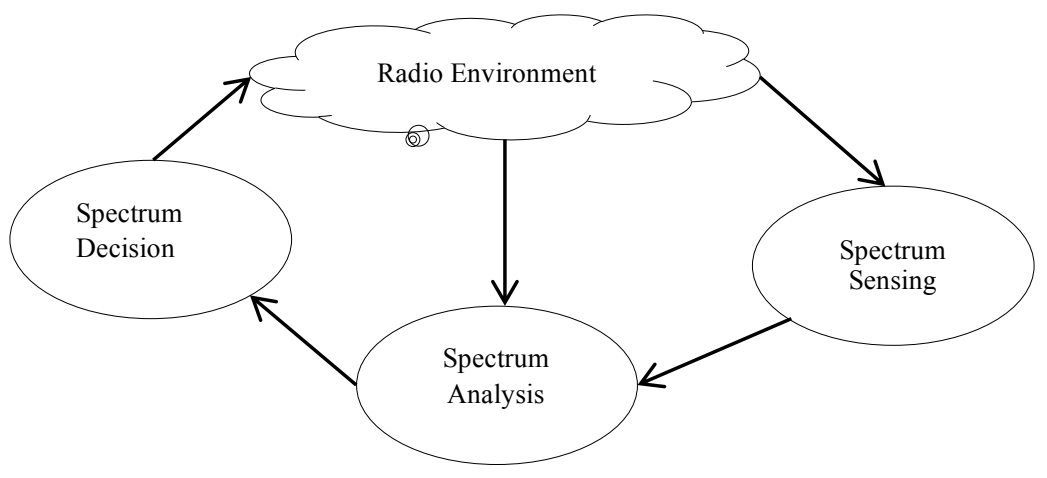

Figure 2. Functions of CR node

\subsection{Spectrum Sensing}

Every node in CR network performs periodical sensing of the available channel state. Each SU node, has two time slots: sensing time and transmission time. In cognitive radio adhoc network, intermediate nodes are involved in data communication between any other two nodes. If any node is in sensing state, virtually the connection is terminated. The data packets received by that node are stored in the buffer. During sensing state, no transmission or reception of data packets take place. This will affect the TCP performance. When the buffer overflows takesplace, there will be packet loss. Also, if intermediate node is in sensing, retransmission timer in sender expires and retransmission of the same data takes place, which leads to spectrum wastage and duplication of packets.

\subsection{Spectrum Changing}

Due to primary user arrival, the secondary user has to move to new channel when the data transmission is proceeding. During spectrum sensing and spectrum changing, virtually the connection gets terminated. These are the main factors that affect the TCP performance during data transmission in CRN. Many different protocols are proposed in the literature to improve the quality-of-service for SU data communication. Briefly the suggested protocol for TCP improvement, various parameters analyzed and comparison among different protocols are seen in the following sections.

\section{Throughput Improvements in Cognitive Radio Network}

Various modifications has been done in the existing TCP protocols such as TCP newReno, TCP Vegas, TCP Westwood etc for having efficient transmission in Cognitive radio network between secondary users. The following are the few works done by different authors to improve throughput by considering various challenging factors in $\mathrm{CRN}$.

\subsection{Modifications based on PU Behaviour in CRN}

In [9], the authors proposed an event driven transport protocol. This protocol is an extension of WTCP in wireless network. TCP Reno protocol is considered for the implementation of PU-Interference handler, faster recovery handler and RTT adjustment function. Throughput was analyzed based on PU activity ratio and bandwidth capacity. Other quality of service such as end-to-end delay, packet loss etc. was not analyzed in this work. This event driven protocol can be extended to a completely mobility based wireless environment. The friendly rate control transport protocol for wired network is analysed in [10]. The authors in [11] proposed an equation based friendly rate control transport protocol for cognitive radio network. PU activity is considered from FCC spectrum database. Data transmission rate is varied based on PU activity in TCP friendly rate control (TFRC) protocol. In this, the throughput and goodput are analyzed. This protocol can be extended for changing spectrum related events instead of PU activity considered from spectrum database.

\subsection{Modifications based on Spectrum Sensing in CRN}

TCP CRAHN implemented by [12] considered the effect of spectrum changing, spectrum sensing and PU behavior. TCP CRAHN is implemented over TCP newReno considering six states of operating modes in cognitive radio network. Six states are connection establishment, normal state, spectrum sensing,

APTIKOM J. CSIT Vol. 3, No. 3, 2018: 106-112 
spectrum change, mobility predicted and route failure. Throughput in normal state is equivalent to the throughput analytical expression of TCP newReno and is given by

$$
\mathrm{B}_{\mathrm{n}}=\frac{\text { DATA }}{\text { RTT }} \sqrt{\frac{3}{2 \mathrm{p}^{\prime}}}
$$

where DATA is the size of the TCP segment, RTT is the round trip time and 'p' is the probability of endto-end packet loss.

In TCP CRAHN, throughput in each time slot is given by

$$
\mathrm{B}=\frac{\mathrm{B}_{\mathrm{n}}\left(1-\mathrm{P}_{\mathrm{s}}-\mathrm{P}_{\mathrm{c}}\right) \mathrm{T}_{\mathrm{p}}+\mathrm{B}_{\mathrm{s}} \mathrm{P}_{\mathrm{s}} \mathrm{s}^{\mathrm{s}}+\mathrm{B}_{\mathrm{c}} \mathrm{P}_{\mathrm{c}} \mathrm{t}_{\mathrm{c}}}{\mathrm{P}_{\mathrm{s}} \mathrm{t}^{\mathrm{s}}+\mathrm{P}_{\mathrm{c}} \mathrm{t}_{\mathrm{c}}+\left(1-\mathrm{P}_{\mathrm{s}}-\mathrm{P}_{\mathrm{c}}\right) \mathrm{T}_{\mathrm{p}}}
$$

where $P_{s}$ and $P_{c}$ are probability that the network is in spectrum sensing state and spectrum changing state respectively. $B_{s}$ and $B_{n}$ are the TCP throughput in the spectrum sensing state and spectrum changing state respectively. Source node modifies the congestion window based on the available bandwidth. This CRAHN throughput analysis performs well but several assumptions are considered related to lower layer parameters [12].

TCP CoBA [13] works in same manner as TCP CRAHN except channel switching. In [13], it is assumed that every secondary users can perform periodic spectrum sensing by using GPS function in it. This protocol updates congestion window (cwnd) based on the bandwidth variations and RTT (Round Trip Time). TCP CoBA receives feedback information from relay nodes inorder to prevent bufferflow and to adjust cwnd size. With respect to fairness index, TCP CoBA outperforms compared to TCP CRAHN. Every node should be interfaced with GPS module to perform well during spectrum sensing is a challenging task.

\subsubsection{Modified Network Coding for TCP}

Due to spectrum sensing, PU behavior and spectrum decision retransmission rate will be high. Inorder to reduce retransmissions, network coding approach is used in [14-15]. In [14], TCP Network Coding-Dynamic Generation Size Adjustment algorithm (TCPNC-DGSA) is used. Here group of packets are coded and is called generation size. Based on generation round trip time (GRTT), the next generation size will be adjusted. This algorithm reduces the retransmissions and improves QoS such as delay and throughput in CRNs. The flow chart of this algorithm is shown in Figure 3.

In [15], the authors have proposed joint generation network coding, where the generation size is adjusted based on the acknowledgement received by the sender node. The flow chart for joint generation network coding is shown in Figure 4. In both [14-15] congestion window adjustment based on the GRTT is not discussed. If there is any packet loss due to spectrum sensing or PU behaviour, window size has to be adjusted. Figure 4 shows the proposed algorithm in [15].

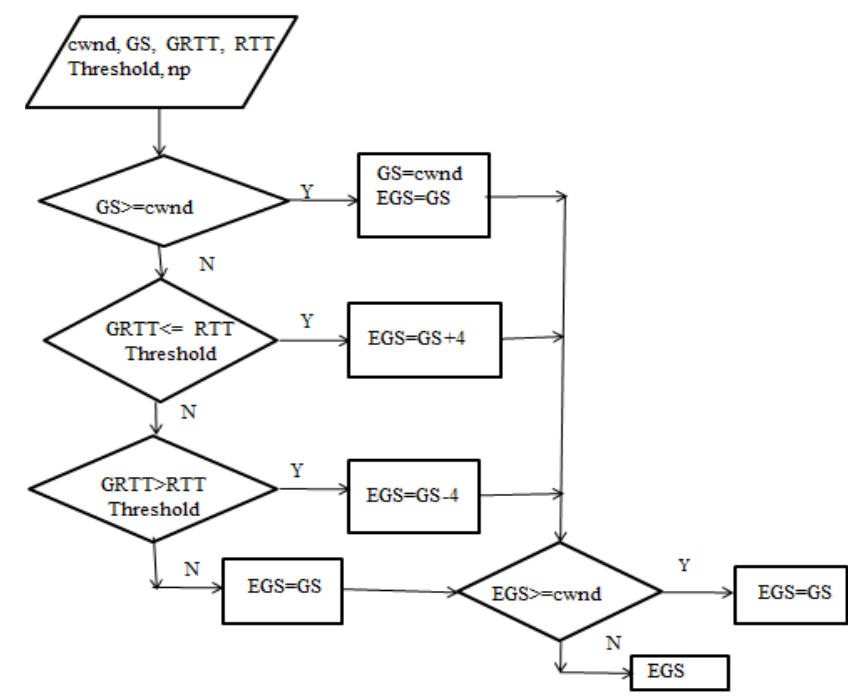

Figure 3. DGSA Algorithm 
where GS: Generation Size, EGS: Next Generation Size, RTT: Round Trip Time.

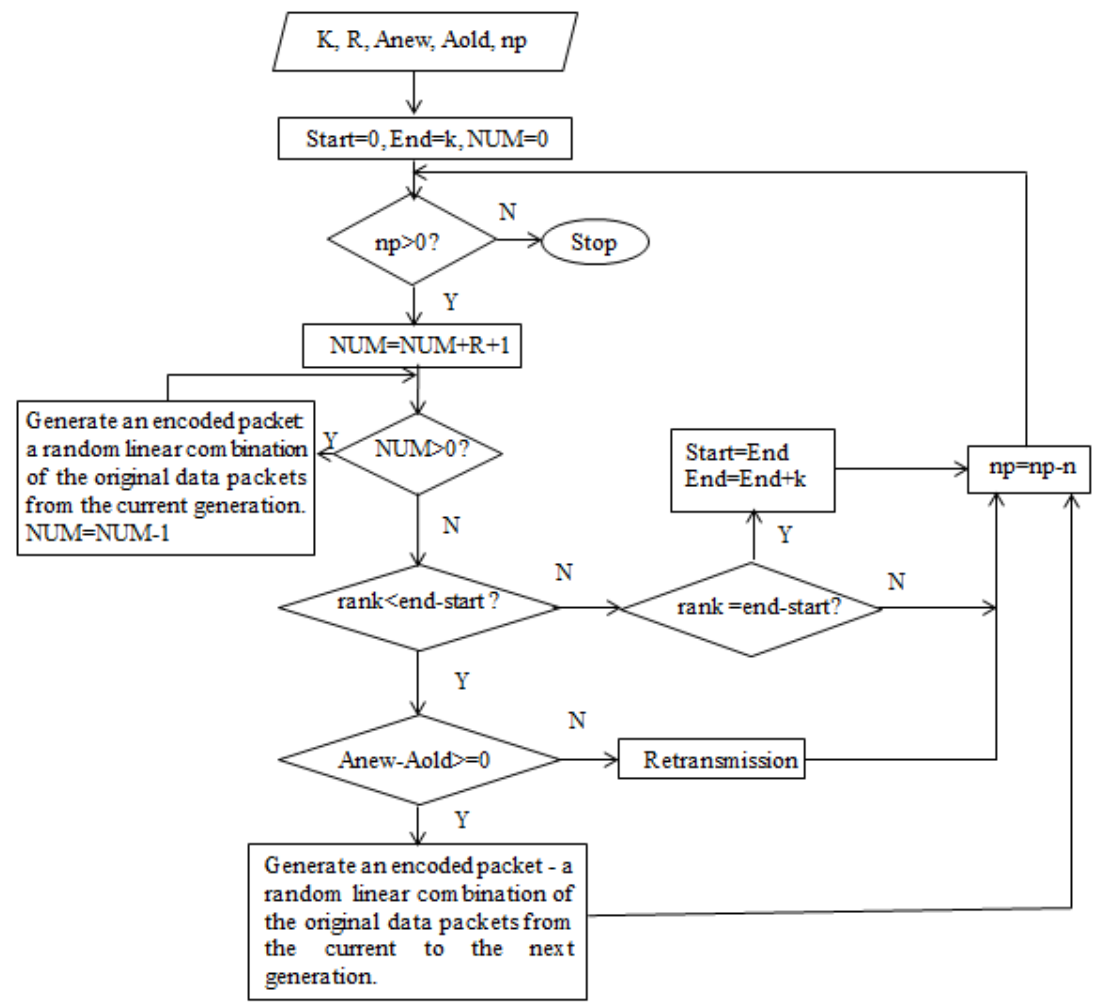

Figure 4. JGNC Algorithm

The joint generation network coding algorithm implemented in [15] is modified according to the sensing characteristics of cognitive radio. It is assumed that in every time slot, each cognitive user performs spectrum sensing before data transmission. During sensing time, the data send to the sensing node will get dropped. The modified joint generation network coding (MJGNC) flow diagram is shown in Figure 5.

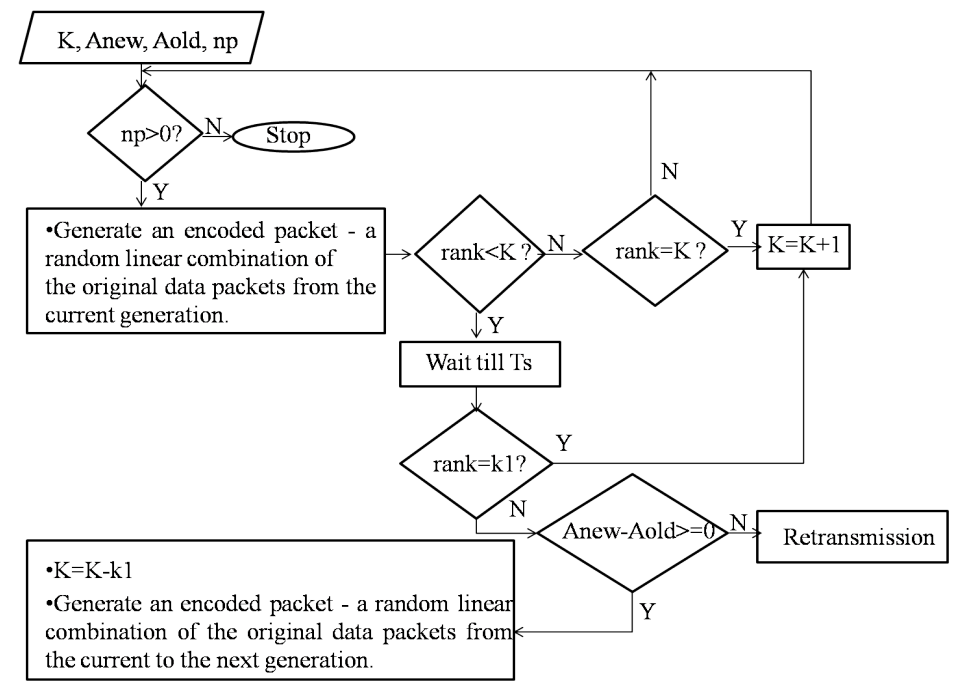

Figure 5. Modified Joint Generation Network Coding

APTIKOM J. CSIT Vol. 3, No. 3, 2018 : 106-112 


\section{Performance Evaluation}

Most of the transport protocols used for cognitive radio network is simulated and verified in NS2, QualNet, NS3 etc. software. The results shown on Figure 6 to Figure 8 are obtained using NS3.The channel bandwidth considered is $2 \mathrm{Mbps}$ and the numbers of channels considered are 3 to 10 . The throughput and end-to-end delay are analyzed. The comparison between DGSA [14] and MJGNC with respect to sensing time and number of packets are shown in Figure 6 to Figure 8. The transport protocol performance in CRN can be optimized by considering the Network layer and MAC layer together. The cross-layer approach can be effectively implemented in NS3 software.

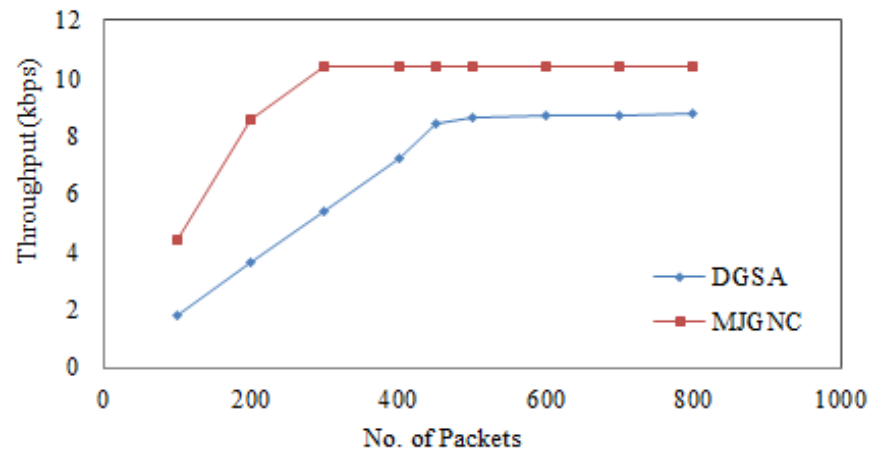

Figure 6. Throughput (kbps) vs No. of Packets

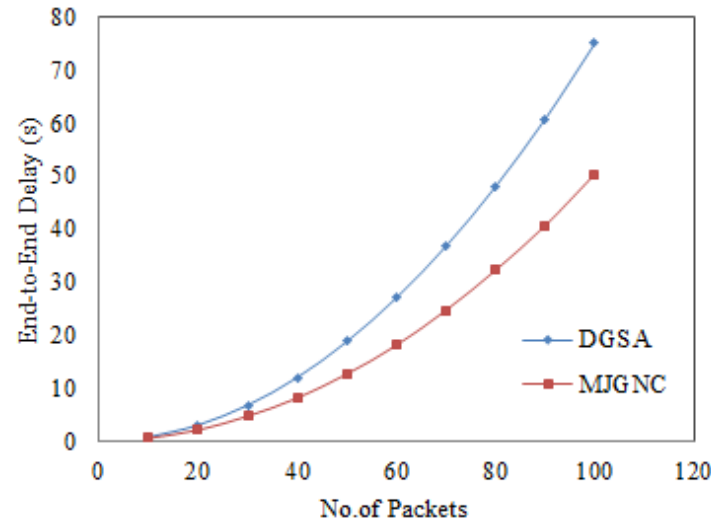

Figure 7. End-to-end Delay(s) vs No. of Packets

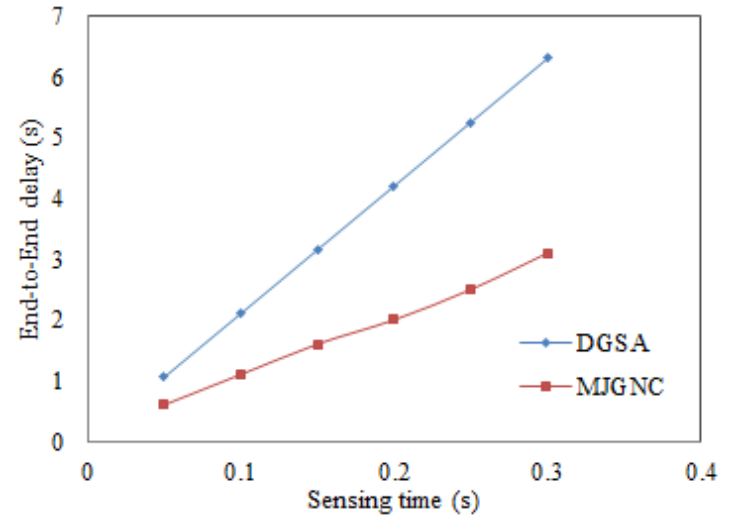

Figure 8. End-to-end Delay(s) vs Sensing time(s)

\section{Conclusion}

This paper gives an insight of various factors that affect the communication of secondary users in cognitive radio network. Many upgradations are done in traditional TCP protocols used in wireless network to perform efficiently in cognitive radio network. The different TCP protocols discussed in section 4 considered some assumptions related to lower layer parameters. To have good improvement in TCP protocol in CRN, the other layers interaction is also required. The future research direction shall include cross-layer design to improve TCP performance for unlicensed user communication.

\section{References}

[1] Mitola, J, Maguire, GQ. Cognitive radio: Making software radios more personal. IEEE Personal Communications. 1999; 6: 13-18,

[2] Akyildiz, IF, Lee, WY, Vuran, MC, Mohanty, S. NeXt generation/ dynamic spectrum access/cognitive radio wireless networks: a survey. Elsevier Computer Networks. 2006; 50: 2127-2159.

[3] Federal Communications Commission, Spectrum policy task force, Technical report. 2000. 
[4] Akyildiz, IF, Lee, WY, Vuran, M, Mohanty, S. A survey on spectrum management in cognitive radio networks. IEEE Communication Magazine. 2008; 46: 40-48.

[5] Anusha M, Srikanth Vemuru, T Gunasekhar. Transmission Protocols in Cognitive Radio Mesh Networks. International Journal of Electrical \& Computer Engineering. 2015; 5(6): 2088-8708.

[6] Ka-Cheong Leung, Victor OK Li. Transmission Control Protocol in Wireless Networks: Issues, Approaches and Challenges. IEEE Communications Surveys. 2006; 8: 64-79.

[7] Allman, M, Paxson, V, Stevens, W. TCP Congestion Control, Request for Comments, RFC 2581. Network Working Group. Internet Engineering Task Force, 1999.

[8] Simon Haykin. Cognitive Radio: Brain-Empowered Wireless Communications. IEEE Journal on selected areas in Communications. 2005; 23: 201-220.

[9] Cheng Yu-Chun, Cheng-Fu Chou, Eric Hsiaokuang Wu, Gen-Huey Chen. A Cognitive TCP Design for a Cognitive RadioNetwork with an Unstable-Bandwidth Link. IEEE Transactions on Computers. 2015; 64: 27302740.

[10] N Ramanjaneya Reddy, Chenna Reddy Pakanati, M Padmavathamma. Performance Enhancement of TCP Friendly Rate Control Protocol over Wired networks. International Journal of Electrical and Computer Engineering (IJECE). 2016; 6(6): 2949-2954.

[11] Abdulla, K, Al-Ali, Kaushik Chowdhury. TFRC-CR: An Equation-based Transport Protocol for Cognitive Radio Networks. Adhoc Networks. 2013; 11: 1836-1847.

[12] Kaushik R Chowdhury, Marco Di Felice, Akyildiz, IF. TCP CRAHN: A Transport Control Protocol for Cognitive Radio Ad Hoc Networks. IEEE Transactions on Mobile Computing. 2013; 12: 790-803.

[13] Kazuya Tsukamoto, Shun Koba, Masato Tsuru, Yuji Oie. Cognitive Radio-Aware Transport Protocol for Mobile Ad Hoc Networks. IEEE Transactions on Mobile Computing. 2015; 14: 288-301.

[14] Xiaoxiong Zhong, Yang Qin, Li Li. TCPNC-DGSA: Efficient Network Coding Scheme for TCP in Multi-hop Cognitive Radio Networks. Wireless Personal Communications. 2015; 84: 1243-1263,.

[15] Yang Qin, Xiaoxiong Zhong, Yuanyuan Yang, Li Li, Fangshan Wu. TCPJGNC: A Transport control protocol based on network coding for multi-hop cognitive radio networks. Computer Communications. 2016; 79: 9 -21.

\section{BIOGRAPHIES OF AUTHORS}

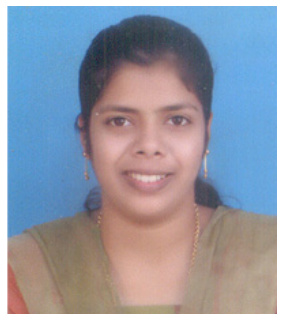

G. Shine Let received M.E degree in Communication Systems from Anna University, Chennai, India in 2007. Currently, she is an Assistant Professor at Karunya University, Coimbatore. Her interests are in Wireless Communication, Mobile Adhoc Network and Cognitive Radio Network.

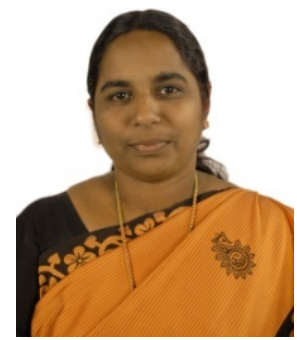

Dr. G. Josemin Bala received M.E degree in Communication Systems from NIT, Trichy, India. She received the Ph.D. degree from Anna University. Currently, she is a professor at Karunya University, Coimbatore. Her research interests include routing algorithms in mobile communication, complexity analysis in VLSI and Image Processing. Dr. Bala has more than 16 years of experience in teaching and research. She supervises five $\mathrm{PhD}$ students in Image Processing, Antenna Design and Networking.

APTIKOM J. CSIT Vol. 3, No. 3, 2018 : 106-112 